\title{
Causas de variación en el fenotipo de la fibrosis quística
}

\author{
Garry R. Cutting \\ Instituto de Medicina Genética, Hospital Johns Hopkins, Baltimore, Md., EE.UU.
}

\section{Palabras clave}

Genes modificadores · Factores ambientales $\cdot$ Gen RTFQ

\section{Extracto}

La causa de la fibrosis quística (FQ) reside en la función anormal del gen regulador de la conductancia transmembránica de la FQ (RTFQ). La FQ es sumamente variable, y mientras algunos pacientes sucumben a la enfermedad durante su primera década, otros viven hasta la cuarta o quinta década. Los pacientes también difieren en la afectación de los sistemas de órganos y en las complicaciones. Una parte de la variación en el fenotipo de la FQ puede atribuirse a la naturaleza del trastorno del gen RTFQ. El fenotipo RTFQ determina fundamentalmente el grado de disfunción exocrina del páncreas y se correlaciona con un grado de anomalía en la concentración de cloruros en el sudor y la malformación del aparato reproductor masculino. No obstante, factores independientes del RTFQ son responsables de la variación en la neumopatía, causa fundamental de morbilidad y mortalidad en la FQ. En el curso de los ultimísimos años se han identificado genes modificadores que influyen sobre la gravedad de la neumopatía en pacientes afectados de FQ. Factores extragenéticos, como el estado socioeconómico y la exposición al tabaquismo pasivo, también afectan el desenlace pulmonar. Estamos asistiendo a un conocimiento más completo de las causas de variación en los pacientes con $\mathrm{FQ}$, lo que facilitará el desarrollo de nuevas medidas pronósticas así como de nuevos tratamientos.

Copyright $\odot 2006$ Nestec Ltd., Vevey/S. Karger AG, Basel (c) 2006 Nestec Ltd., Vevey/S. Karger AG, Basel 0252-8185/06/0643-0111\$23.50/0

Fax +41613061234 E-Mail karger@karger.ch www.karger.com
Accesible online en: www.karger.com/ans

\section{Introducción}

La fibrosis quística (FQ), un trastorno monogénico que afecta a 60.000 sujetos en todo el mundo, está causada por trastornos del gen regulador de la conductancia transmembránica de la FQ (RTFQ). Se observa fundamentalmente en individuos de ascendencia blanca europea y con una incidencia comprendida entre 1:2.000 y 1:4.000 [1]. La FQ es mucho menos corriente en poblaciones africanas y asiáticas nativas. Algunos grupos de raza blanca presentan una mayor frecuencia de FQ que otros, probablemente debido a un efecto de descubrimiento [2, $3]$. Aunque se desconoce la causa de la elevada frecuencia de portadores de mutaciones de FQ en la raza blanca (1 de 30), la superioridad heterocigótica es la explicación preferida [1]. Cualquier mecanismo implicado tiene que tener en cuenta que la FQ en la raza blanca se debe a la presencia de una mutación causante de FQ común, que está ausente en otras razas excepto en casos atribuibles a mezclas. Por lo tanto, la explicación de la elevada frecuencia de la mutación común ( $\Delta \mathrm{F} 508$; ver más adelante) también explicará probablemente la frecuencia de la FQ entre las personas blancas. Ahora que conocemos la etiología de la FQ, el reto actual consiste en determinar por qué los pacientes presentan desenlaces variables. Esta revisión aporta un resumen de nuestro conocimiento actual del papel de la variación en el RTFQ y presenta pruebas crecientes de la importancia de factores independientes del RTFQ en la determinación de la gravedad de la enfermedad en pacientes afectados de FQ. 


\section{Variación en el fenotipo de la FQ}

Cuando en la década de los 40 la FQ se describió por vez primera como entidad bien diferenciada, la mayoría de los pacientes fallecía a causa de esta enfermedad antes de su primer aniversario debido a la malnutrición causada por una disfunción del páncreas exocrino. El tratamiento de la insuficiencia pancreática exocrina, en particular el desarrollo del tratamiento sustitutivo de enzimas, permitió que los pacientes sobrevivieran más allá de su primera década. La mediana de supervivencia ha aumentado progresivamente desde los años $40 \mathrm{y}$, en la actualidad, los pacientes afectados de FQ presentan una mediana de esperanza de vida de 36,2 años en los Estados Unidos. A pesar de los incrementos impresionantes de la mediana de supervivencia, siguen existiendo amplias variaciones en la edad en el momento de la muerte de los pacientes con FQ. Algunos de estos pacientes fallecen antes de su décimo cumpleaños, mientras que otros viven hasta las décadas de los 50 y 60 años. La neumopatía es actualmente la causa principal de morbilidad y mortalidad en la FQ, así como la causa del $90 \%$ de todas las muertes ocasionadas por la FQ [4]. La gravedad de la neumopatía varía considerablemente entre los pacientes con FQ. Por ejemplo, algunos pacientes desarrollan una pauta obstructiva en los primerísimos años de vida, complicada por una infección causada por Pseudomonas aeruginosa, microorganismos fúngicos y, posteriormente en el curso de la vida, micobacterias atípicas. Otros pacientes experimentan síntomas relativamente escasos de neumopatía hasta la década de los 20 años, momento en que aparece un episodio de hemoptisis seguido de un rápido declive que obliga a un trasplante de pulmón en el curso de unos pocos años. La mayoría de los pacientes con FQ presenta también rinosinusitis crónica y un subgrupo de pacientes, poliposis nasal.

En casi todos los pacientes afectados de FQ se presentan anomalías pancreáticas. La mayoría de pacientes sufre deficiencia exocrina grave, que exige un aporte complementario de enzimas para lograr una absorción suficiente de grasas e hidratos de carbono y la prevención de deficiencias vitamínicas. Una pequeña proporción de pacientes con FQ (5 a 10\%) presentan una absorción suficiente de grasas e hidratos de carbono sin necesidad de aportes complementarios de enzimas. Estos son considerados pancreáticamente suficientes. Este último grupo de pacientes presenta habitualmente un curso más leve de la enfermedad con neumopatía menos grave, anomalías más leves de la concentración de cloruros en el sudor y menos complicaciones gastrointestinales [5]. No obstan- te, algunos muestran signos de neumopatía grave manifiesta que obliga a realizar un trasplante de pulmón. El páncreas endocrino está también afectado en la FQ. Dado que la mayoría de los pacientes con FQ sobrevive en la edad adulta, se ha comprobado que una proporción importante presenta intolerancia a la glucosa y que el $30 \%$ de los pacientes con FQ son diagnosticados de diabetes en torno a los 35 años de edad [6]. La aparición de diabetes se ha relacionado con una neumopatía más grave y una reducción de la longevidad [7].

Entre las demás manifestaciones gastrointestinales de la FQ destacan hepatopatía y obstrucción intestinal. La mayoría de los pacientes con FQ presenta cierto grado de enfermedad hepatobiliar en el momento de la autopsia, y un subgrupo de pacientes (alrededor del 5\%) sufre hepatopatía grave con cirrosis, hipertensión portal y varices esofágicas [8]. La aparición de estas características se asocia habitualmente a un acortamiento de la supervivencia; alrededor del $2 \%$ de los pacientes fallecen debido a complicaciones asociadas a la hepatopatía [4]. La obstrucción intestinal durante el periodo neonatal, denominada íleo meconial (IM), es un cuadro muy bien identificado que afecta aproximadamente al $15 \%$ de los pacientes con FQ [4]. Las modernas técnicas quirúrgicas pediátricas permiten que esta complicación sea mortal sólo en los casos más extremos. En algunas situaciones, la obstrucción puede aliviarse con un enema de gastrografin. El síndrome de obstrucción intestinal distal (SOID) afecta a niños mayores y adultos con FQ. Se ha estimado que del 7 al $40 \%$ de los pacientes afectados por la FQ experimentan un episodio de SOID durante su vida [4]. IM y SOID presentan rasgos clínicos similares, habiéndose descrito que los pacientes con IM presentan una mayor tasa de SOID, lo que permite suponer que comparten una etiología común.

La elevación de la concentración de sodio y cloruros en el sudor es una característica bioquímica bien diferenciada de la FQ. La anomalía de la concentración de cloruros tiene una mayor especificidad con respecto a la FQ, y se ha utilizado como herramienta diagnóstica durante más de cuatro décadas. El margen de concentraciones de cloruros en el sudor varía entre las personas normales y los pacientes con FQ. Algunos adultos sanos pueden presentar concentraciones de cloruros en el sudor comprendidas entre 40 y $60 \mathrm{mmol} / \mathrm{l}$. No obstante, esta concentración de cloruros en el sudor de un lactante o niño pequeño es coherente con el diagnóstico de FQ. La mayoría de los pacientes con FQ presenta cloruros sudorales $>60$ $\mathrm{mmol} / \mathrm{l}$, con un promedio de unos $105 \mathrm{mmol} / \mathrm{l}$. En una pequeña proporción de pacientes afectados de $\mathrm{FQ}$, las 
anomalías de la concentración iónica del sudor pueden ocasionar una pérdida de sal excesiva, así como deshidratación hiponatrémica.

La inmensa mayoría de hombres con FQ (98\%) es infértil debido a anomalías en el desarrollo de estructuras derivadas del conducto de Wolf. Entre éstas destacan la malformación o la ausencia del vas deferens y anomalías de las vesículas seminales. También se ha descrito que la motilidad y la morfología de los espermatozoides son anormales; no obstante, espermatozoides adquiridos por aspiración testicular han generado concepciones viables.

\section{Contribución del genotipo RTFQ}

El gen RTFQ codifica una proteína de 1.480 aminoácidos, que funciona como un canal de cloruros regulado por ANPc y como regulador de otros canales iónicos. El $R T F Q$ es responsable de regular el trasporte de iones y líquido a través de las membranas apicales de las células epiteliales, y desempeña un papel primordial en la absorción y secreción en el revestimiento epitelial de las vías respiratorias pulmonares y los conductos pancreáticos. La disfunción del RTFQ causa alteraciones en el contenido líquido y salino del líquido de la superficie de las vías respiratorias y las secreciones pancreáticas, dando lugar a un deterioro de los mecanismos defensivos y a obstrucción que, en última instancia, destruyen ambos órganos. El Consorcio de Análisis Genéticos de la FQ (www.genet. sickkids.on.ca/cftr) ha recibido notificaciones de más de 1.400 mutaciones en el gen RTFQ; de estas mutaciones, 1.200 se han asociado a la enfermedad mientras que las 200 restantes parecen corresponder a variantes benignas. A pesar de esta diversidad alélica extrema, una de las mutaciones, una deleción de fenilalanina en la posición 508 (denominada $\Delta \mathrm{F} 508$ ) representa el $70 \%$ de los alelos de la FQ en todo el mundo [10]. La mutación $\Delta$ F508 se encuentra casi exclusivamente en blancos europeos y aparece con frecuencia creciente desde la cuenca mediterránea hasta las regiones septentrionales de Europa [10]. Aproximadamente 5 a 20 de otras mutaciones del RTFQ representan del 10 al 20\% de los alelos de FQ [10]. La distribución de las mutaciones 'menos corrientes' varía en función de la población. El resto de las mutaciones de RTFQ es raro, con excepción de un puñado que ha mostrado una frecuencia elevada en poblaciones aisladas debido a un efecto de descubrimiento. La consecuencia de la elevada frecuencia de la mutación $\Delta$ F508 radica en que la mitad de todos los pacientes con FQ comparten el mismo genotipo $R T F Q$ (es decir $\Delta \mathrm{F} 508 / \Delta \mathrm{F} 508$ ). Los homocigotos $\Delta \mathrm{F} 508$ han sido estudiados extensamente, comprobándose que este genotipo se asocia casi invariablemente a insuficiencia pancreática [11]. Estos pacientes presentan elevadas concentraciones de cloruros en el sudor, y todos los varones con este genotipo son infértiles. No obstante, la gravedad de la neumopatía en los homocigotos $\Delta \mathrm{F} 508$ varía considerablemente $[11,12]$.

El extenso conjunto de pacientes afectados de FQ con un genotipo $R T F Q$ idéntico (homocigosidad $\triangle \mathrm{F} 508$ ) proporciona una población de referencia para evaluar el efecto de otras mutaciones del RTFQ sobre el fenotipo FQ. Estudios de genotipo/fenotipo han revelado que el genotipo RTFQ se correlaciona en gran medida con la gravedad de la insuficiencia pancreática [11]. Aunque la mayoría de las mutaciones se asocia a insuficiencia pancreáti$\mathrm{ca}$, existen alrededor de dos docenas de mutaciones asociadas a suficiencia pancreática. Estas mutaciones deterioran pero no suprimen la función del RTFQ [13]. Por lo tanto, la función pancreática residual parece consecuencia de la función parcial del RTFQ. No obstante, esta asociación no es absoluta; algunos pacientes portadores de mutaciones que permiten cierta función del RTFQ sufren insuficiencia pancreática [13]. Existen también mutaciones que causan anomalías específicas de la función pancreática, como la mutación L997F, que se asocia en gran medida a pancreatitis [14]. Las complicaciones en el tubo digestivo, como el IM, suelen aparecer en pacientes con mutaciones causantes de insuficiencia pancreática. No obstante, los pacientes portadores de la mutación G551D, una mutación que causa insuficiencia pancreática, presentan una menor tasa de IM en comparación con los pacientes homocigotos $\Delta$ F508 $[15,16]$. Si bien la suficiencia pancreática ha sido asociada a una neumopatía menos grave, ha resultado difícil hallar mutaciones específicas que confieran tanto suficiencia pancreática como neumopatía leve. Uno de los ejemplos de esto último es la mutación A455E. En dos estudios se ha demostrado que los pacientes con FQ portadores de A455E presentan una progresión más lenta de su neumopatía que los pacientes homocigóticos para la mutación $\Delta \mathrm{F} 508$ [17, 18].

El genotipo $R T F Q$ se correlaciona moderadamente con el grado de anomalía de cloruros en el sudor, lo cual aparece generalmente en el contexto de una suficiencia pancreática. En otras palabras, los pacientes portadores de mutaciones con función parcial que confiere suficiencia pancreática, tienden también a presentar elevaciones menos anormales de la concentración de cloruros en el sudor [19]. Por último, las anomalías del aparato reproductor de los varones afectados de FQ guardan una rela- 
ción estrecha con el genotipo RTFQ. La mutación de empalme $3849+10 \mathrm{kbC} \rightarrow \mathrm{T}$ se asocia a una tasa elevada de fertilidad en varones con FQ a pesar de causar signos pulmonares coherentes con FQ [20].

Dos mutaciones RTFQ demuestran hasta que punto puede ser sutil la asociación entre genotipo y fenotipo. Una de las mutaciones que causa FQ $(\mathrm{R} 117 \mathrm{H})$ es también una mutación corriente en hombres con ausencia bilateral congénita aislada del vas deferens (ABCVD). Este último proceso presenta características idénticas a las halladas en hombres con FQ; sin embargo, hombres con ABCVD no presentan neumopatía potencialmente mortal incluso cuando son portadores de mutaciones en cada gen RTFQ. La diferencia entre hombres con FQ y hombres con $\mathrm{ABCVD}$, ambos portadores de la mutación R117H, es que los primeros presentan una segunda mutación en el gen [21]. En consecuencia, la presencia de una segunda alteración (denominada 5T), que reduce la cantidad de proteína RTFQ producida, da lugar a que los hombres con ABCVD presenten también neumopatía potencialmente mortal. De manera intrigante, $5 \mathrm{~T}$ causa la enfermedad FQ por sí misma cuando se asocia a una anomalía de la longitud de una variante secuencial adyacente denominada tracto TG. Los varones que presentan un tracto TG más prolongado, asociado a la variante $5 \mathrm{~T}$, y otra mutación en el gen $R T F Q$ (como $\triangle \mathrm{F} 508$ ), suelen tener ABCVD. Aquéllos con la misma combinación de mutaciones (por ejemplo, $\Delta$ F508 y $5 \mathrm{~T}$ ) que presentan el tracto TG corto, más corriente, no presentan habitualmente anomalías [22]. Esta asociación entre genotipo RTFQ y fenotipo FQ requiere que las múltiples alteraciones en $R T F Q$ se consideren contributivas al desenlace.

\section{Contribución de modificadores genéticos}

Aunque se dispone de algunas pruebas de la asociación entre mutaciones de FQ y neumopatía (por ejemplo, A455E y R117H, descritos anteriormente), en la mayoría de las ocasiones el genotipo RTFQ no pronostica la gravedad de la neumopatía. Esto es debido fundamentalmente a la amplia variabilidad de la función pulmonar observada en pacientes con FQ que presentan genotipos $R T F Q$ idénticos [12]. Esta observación indica que factores independientes del RTFQ deben contribuir considerablemente a la variación en la neumopatía.

Uno de los métodos más eficaces para determinar el papel de factores genéticos en la variabilidad patológica en humanos consiste en estudiar familias con múltiples niños afectados. Los hermanos comparten el 50\% de sus genes. Por lo tanto, la recurrencia de complicaciones entre hermanos, con una tasa superior a la observada en pacientes sin parentesco, puede implicar factores genéticos. Un ejemplo es el IM. Si el primer hermano en una familia presenta FQ e IM, cada hermano subsiguiente con FQ presenta un riesgo del 25\% de presentar también IM. El riesgo de recurrencia para los hermanos es considerablemente superior que en pacientes sin parentesco (alrededor del 15\%). Otros ejemplos de complicaciones con grandes riesgos de recurrencia y características patológicas con elevada similitud entre hermanos comprenden la función pancreática, la hepatopatía y las infecciones causadas por Pseudomonas [23-26]. No obstante, dado que los hermanos comparten también su entorno (hogar, escuela, clínica, etc.), las tasas de recurrencia más elevadas entre hermanos no implican necesariamente modificadores genéticos. Un método formal para verificar el efecto de modificadores genéticos frente a modificadores no genéticos consiste en estudiar a gemelos afectados. La comparación de gemelos monocigóticos (100\% de genes compartidos) y dicigóticos (50\% de genes compartidos) permite efectuar estimaciones de los efectos genéticos (es decir, la herencia). Basándose en este abordaje, investigadores han demostrado que una medida de la masa corporal (talla para peso) está determinada fundamentalmente por genes [27]. También parece que factores genéticos independientes del RTFQ influyen sobre la función intestinal en pacientes con FQ, alterando la secreción de cloruros $[28,29]$ y pudiendo modificar el crecimiento en pacientes afectados de FQ [30].

Uno de los métodos para identificar genes específicos que modifican el fenotipo FQ es seleccionar genes candidatos basándose en el conocimiento de la fisiopatología de la FQ. Estos genes candidatos codifican proteínas que desempeñan un papel en la progresión de la enfermedad (por ejemplo, mediadores inflamatorios). Se identifican las variantes de $\mathrm{ADN}$ en estos genes candidatos y se determina la distribución de las variantes de $\mathrm{ADN}$ en los pacientes con FQ estratificados con arreglo a la gravedad de la enfermedad. El descubrimiento de que variantes de un gen candidato se asocian a una enfermedad más grave confiere al candidato el papel de modificador. Si bien este enfoque es poderoso, conlleva también la carga del problema inherente a la división de los pacientes en grupos diferentes. Las variantes genéticas pueden diferir por azar o debido a diferencias no identificadas entre ambos grupos, lo que afecta a la distribución de variantes que no se correlacionan con la gravedad de la enfermedad. En consecuencia, los estudios de asociación de casos y controles tienden a generar un cierto número de genes mo- 
dificadores candidatos falsos positivos. Uno de los abordajes que reduce los falsos positivos consiste en examinar genes candidatos en diferentes poblaciones de pacientes. Basándose en este enfoque, Drumm y cols. [31] investigaron 10 genes candidatos en los que se había demostrado previamente que modificaban la gravedad de la enfermedad en pacientes con FQ, en un estudio multicéntrico en 808 pacientes afectados de $\mathrm{FQ}$, reclutados basándose en la gravedad de la neumopatía. De estas 10 variantes genéticas, sólo uno de los genes 'modificadores' (factor de crecimiento transformador- $\beta 1$, TGF $\beta 1$ ) se asociaba a una neumopatía más grave. Los autores confirmaron sus datos investigando el gen TGF $\beta 1$ en un segundo grupo de 498 pacientes con FQ [31]. Variantes en el gen TGF $\beta 1$ han sido relacionadas con una propensión al asma y protección frente al desarrollo de neumopatía obstructiva crónica en fumadores, respaldando el concepto de que los genes modificadores de la FQ pueden desempeñar también un papel patológico en enfermedades corrientes [32, 35]. Variantes de otros dos genes (factor de necrosis tumoral- $\alpha$ y lectina que se une a manosa) han sido asociados a la gravedad de la neumopatía de la FQ, con independencia del genotipo $R T F Q$, en más de un grupo de pacientes afectados de FQ [36-40]. Estos dos genes se incluyeron en el estudio de replicación de Drumm y cols. [31] sin que mostraran asociación alguna a la gravedad de la neumopatía. No obstante, en cualquier estudio, los genes modificadores que producen un efecto real aunque escaso pueden ser difíciles de detectar. Efectos modificadores sutiles sobre el fenotipo pueden quedar enmascarados por la variación debida a factores extragenéticos, como una diferencia en el tratamiento entre centros [41]. En consecuencia, no debe sorprender que cierto número de otros genes modificadores candidatos hayan mostrado asociación en sólo uno de los estudios o no hayan mostrado asociación coherente alguna a la gravedad de la enfermedad [42].

En el curso de los próximos años, es probable que nuevos estudios confirmen o refuten el papel de cierto número de genes en la modificación del fenotipo FQ. Además, se prevé que otros abordajes para identificar genes modificadores, como la clonación posicional, revelen genes que no hubieran sido seleccionados basándose en el enfoque de los genes candidatos. Estos genes podrían tener funciones diferentes de las contempladas para los modificadores, aunque, en virtud de su correlación genética con la enfermedad, podría demostrarse su papel de modificadores de la FQ. Este enfoque fue utilizado para identificar el RTFQ como el gen responsable de la FQ. Los estudios basados en familias son sumamente adecuados para este último enfoque. Los hermanos afectados de FQ pueden agruparse con arreglo al grado de similitud con respecto a una medida de la gravedad de la enfermedad (por ejemplo, función pulmonar). A continuación, se verifican en los hermanos las correlaciones entre el grado de compartimiento de una región genética y el grado de similitud en cuanto a la gravedad de la enfermedad. Por lo tanto, en hermanos que presentasen una neumopatía grave se investigaría el compartimiento de genes modificadores capaz de causar la neumopatía grave. No se esperaría que hermanos diferentes, uno con enfermedad grave y otro con enfermedad leve, presentasen un gen modificador común. Este método, denominado concordancia, ha sido aplicado en hermanos con FQ para demostrar el papel de una región en el cromosoma 19 que pudiera contener un modificador para el IM [43]. Una ampliación eficaz de los métodos basados en familias consiste en investigar a padres y a su descendencia afectada de FQ con respecto a la transmisión de genes modificadores. En esta situación se determina si una variante de un gen modificador es transmitido más frecuentemente de lo previsto por azar a descendientes agrupados con arreglo a la gravedad de la enfermedad. Este método, denominado prueba de desequilibrio de transmisión (PDT), no está sujeto a errores debidos a la estratificación de la población, que afectan a los estudios de asociación. No obstante, la PDT debe aplicarse a ambos padres para conseguir una eficacia máxima. La obtención del ADN de todos los miembros de la familia puede ser difícil, especialmente en el caso de pacientes más mayores. Sin embargo, los estudios basados en familias en Europa, Canadá y EE.UU. incluyen colecciones de pacientes idóneos para la PDT.

\section{El papel de los modificadores ambientales}

La mejoría de la supervivencia de los pacientes con $\mathrm{FQ}$ durante las últimas cinco a seis décadas no puede atribuirse a genes modificadores. Los cambios en el tratamiento, especialmente el desarrollo del tratamiento sustitutivo de enzimas pancreáticas y la creación de centros multidisciplinarios para la asistencia de la FQ, ha propiciado cambios considerables en el desenlace de los pacientes con FQ (ver el capítulo de B. Strandvik, 'Asistencia de pacientes con fibrosis quística', en este número). Estos 'factores modificadores' son de naturaleza ambiental. La identificación de exposiciones ambientales específicas que afectan al desenlace proporciona un método eficaz para hallar factores que podrían ser manipulados fácilmente. La evaluación de la contribución ambiental al fe- 
Fig. 1. Causas de variación en las manifestaciones principales de la FQ. Se estima la contribución relativa del genotipo RTFQ, los genes modificadores y los factores ambientales para cada uno de los sistemas de órganos afectados en los pacientes con FQ. El genotipo RTFQ es el determinante principal de la gravedad de la enfermedad pancreática y la malformación de las estructuras reproductoras masculinas. Los genes modificadores y los factores ambientales desempeñan papeles importantes en la gravedad de la neumopatía.

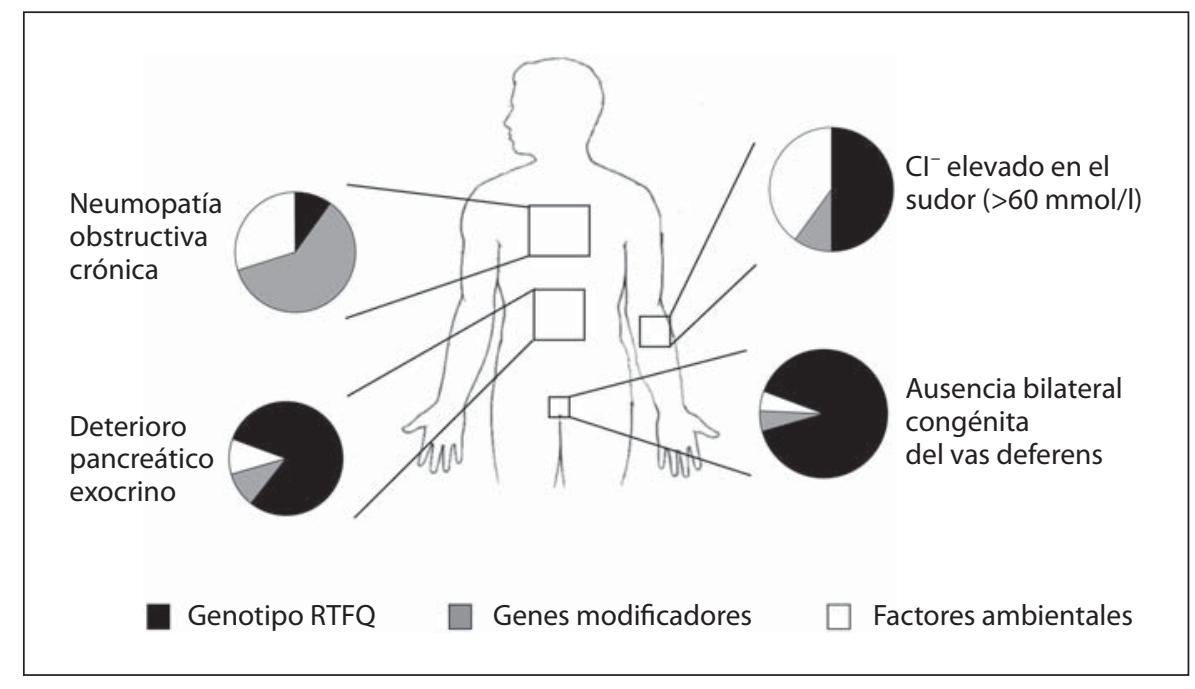

notipo ha sido problemática debido a la variedad de los componentes implicados y a la falta de mediciones objetivas de numerosos componentes. A pesar de estas limitaciones, el consumo pasivo de cigarrillos se ha relacionado con una función pulmonar menoscabada [44-46]. La eliminación del tabaquismo pasivo de los pacientes con FQ es una medida relativamente simple para mejorar el desenlace. También se ha comprobado que la intervención nutricional agresiva afecta al desenlace [47-50]. Es interesante destacar que los pacientes varían en su respuesta al tratamiento nutricional, lo que permite suponer que otros factores ambientales y/o genéticos pueden desempeñar un papel [51]. La colonización pulmonar con $P$. aeruginosa y Burkholderia cepacia es un acontecimiento de mediación ambiental $[52,53]$, que se asocia a la reducción de la longevidad [54-57]. Nuevamente, los cambios en los factores ambientales, como el control de las infecciones, pueden evitar la difusión de subtipos virulentos de estas bacterias.

La mejoría del desenlace por reducción de la variación en el tratamiento entre los centros asistenciales, de manera que todos los pacientes reciban una asistencia optimizada, es una piedra angular del tratamiento actual de la FQ. Las fundaciones de apoyo a pacientes han utilizado sus recursos y capacidades organizativas para desarrollar centros asistenciales multidisciplinarios que permitan suministrar niveles de asistencia óptimos y uniformes. Los esfuerzos recientes en EE.UU. se han orientado a mejorar el estado nutricional de los pacientes con $\mathrm{FQ}$, basándose en el conocimiento de que un estado nutricional deficiente se asocia a una neumopatía y a un desenlace más desfavorable. Por último, el cumplimiento es evidentemente una cuestión de gran importancia para los pacientes con FQ, lo mismo que para los pacientes afectados de cualquier trastorno crónico. Aún cuando se ha desarrollado una terapia capaz de alterar la función de la proteína RTFQ, el tratamiento satisfactorio podría depender de la buena voluntad del paciente para seguir una pauta terapéutica vitalicia. En consecuencia, es probable que el énfasis sobre las conductas de bienestar y la adherencia a la asistencia sistemática y al tratamiento previsor genere mejoras constantes del desenlace de los pacientes con FQ.

\section{Resumen}

Se ha observado un éxito sorprendente en el tratamiento de la $\mathrm{FQ}$, un proceso que al principio era frecuentemente mortal en el curso del primer año de vida. La identificación de los factores clave que determinan el desenlace en el paciente individual y el desarrollo del tratamiento que aborda dichos factores están mejorando la longevidad de todos los pacientes afectados de FQ. La identificación del gen $R T F Q$ representó un paso clave en el conocimiento de la fisiopatología a nivel molecular y en la determinación del grado en el cual la variación en la función del RTFQ influye sobre el desenlace de la FQ. Estos estudios revelaron que otros modificadores, tanto genéticos como extragenéticos, desempeñan un papel importante en la determinación de la gravedad de la enfermedad (fig. 1). El reto para la próxima década consistirá en identificar y caracterizar esos factores, de manera que pueda optimizarse el desenlace de todos los pacientes afectados de FQ. 


\section{Bibliografía}

1 Cutting GR: Cystic fibrosis; in Emery and Rimoin's Principles and Practice of Medical Genetics, ed 4. London, Churchill-Livingston, 2002.

-2 Fujiwara TM, Morgan K, Schwartz RH, et al: Genealogical analysis of cystic fibrosis families and chromosome 7q RFLP haplotypes in the Hutterite Brethren. Am J Hum Genet 1989;44:327-337.

$>3$ Super M: Cystic fibrosis in the South West African Afrikaner. An example of population drift, possibly with heterozygote advantage. S Afr Med J 1975;49:818-820.

4 Cystic Fibrosis Foundation: Cystic Fibrosis Foundation Patient Registry Annual Data Report 1999. Bethesda, Cystic Fibrosis Foundation, 2000.

$>5$ Gaskin KJ, Gurwitz D, Durie P, et al: Improved respiratory prognosis in patients with cystic fibrosis with normal fat absorption. J Pediatr 1982;100:857-862.

6 Cystic Fibrosis Foundation: Cystic Fibrosis Foundation Patient Registry Annual Data Report 2004. Bethesda, Cystic Fibrosis Foundation, 2004

7 Marshall BC, Butler SM, Stoddard M, et al: Epidemiology of cystic fibrosis-related diabetes. J Pediatr 2005;146:681-687.

8 di Sant'Agnese PA, Hubbard VA: The hepatobiliary system; in Taussig LM (ed): Cystic Fibrosis. New York, Thieme Stratton, 1984, pp 296-322.

$>9$ Dray X, Bienvenu T, Desmazes-Dufeu N, et al: Distal intestinal obstruction syndrome in adults with cystic fibrosis. Clin Gastroenterol Hepatol 2004;2:498-503.

$>10$ Bobadilla JL, Macek M, Fine JP, Farrell PM: Cystic fibrosis: a worldwide analysis of CFTR mutations - correlation with incidence data and application to screening. Hum Mutat 2002; 19:575-606.

11 Kerem E, Corey M, Kerem B-S, et al: The relation between genotype and phenotype in cystic fibrosis-analysis of the most common mutation (deltaF508). N Engl J Med 1990; 323:1517-1522.

12 Cystic Fibrosis Genotype-Phenotype Consortium: Correlation between Genotype and Phenotype in Patients with Cystic Fibrosis. N Engl J Med 1993;329:1308-1313.

13 Koch C, Cuppens H, Rainisio M, et al: European Epidemiologic Registry of Cystic Fibrosis (ERCF): comparison of major disease manifestations between patients with different classes of mutations. Pediatr Pulmonol 2001;31:1-12.

-14 Gomez LM, Benetazzo MG, Marzari MG, et al: High frequency of cystic fibrosis transmembrane regulator mutation $\mathrm{L} 997 \mathrm{~F}$ in patients with recurrent idiopathic pancreatitis and in newborns with hypertrypsinemia. Am J Hum Genet 2000;66:2013-2014.
15 Hamosh A, King TM, Rosenstein BJ, et al: Cystic fibrosis patients bearing the common missense mutation Gly $\rightarrow$ Asp at codon 551 and the deltaF508 are indistinguishable from deltaF508 homozygotes except for decreased risk of meconium ileus. Am J Hum Genet 1992;51:245-250.

16 Feingold J, Guilloud-Bataille M: Genetic comparisons of patients with cystic fibrosis with or without meconium ileus. Clinical Centers of the French CF Registry. Ann Genet 1999;42:147-150.

17 Gan KH, Veeze HJ, van den Ouweland AMW, et al: A cystic fibrosis mutation associated with mild lung disease. N Engl J Med 1995;333:95-99.

-18 De Braekeleer M, Allard C, Leblanc J-P, et al: Genotype-phenotype correlation in cystic fibrosis patients compound heterozygous for the A455E mutation. Hum Genet 1997;101: 208-211.

19 Wilschanski M, Zielenski J, Markiewicz D, et al: Correlation of sweat chloride concentration with classes of the cystic fibrosis transmembrane conductance regulator gene mutations. J Pediatr 1995;127:705-710.

20 Highsmith WE Jr, Burch LH, Zhou Z, et al: A novel mutation in the cystic fibrosis gene in patients with pulmonary disease but normal sweat chloride concentrations. N Engl J Med 1994;331:974-980.

21 Kiesewetter S, Macek M Jr, Davis C, et al: A mutation in the cystic fibrosis transmembrane conductance regulator gene produces different phenotypes depending on chromosomal background. Nat Genet 1993;5:274278.

22 Groman JD, Hefferon TW, Casals T, et al: Variation in a repeat sequence determines whether a common variant of the cystic fibrosis transmembrane conductance regulator gene is pathogenic or benign. Am J Hum Genet 2004;74:176-179.

23 Corey M, Durie P, Moore D, et al: Familial concordance of pancreatic function in cystic fibrosis. J Pediatr 1989;115:274-277.

24 Santis G, Osborne L, Knight RA, Hodson $\mathrm{ME}$ : Independent genetic determinants of pancreatic and pulmonary status in cystic fibrosis. Lancet 1990;336:1081-1084.

25 Duthie A, Doherty DG, Williams C, et al: Genotype analysis for deltaF508, G551D and R553X mutations in children and young adults with cystic fibrosis with and without liver disease. Hepatology 1992;15:660-664.

26 Picard E, Aviram M, Yahav Y, et al: Familial concordance of phenotype and microbial variation among siblings with CF. Pediatr Pulmonol 2004;38:292-297.

27 Mekus F, Ballmann M, Bronsveld I, et al: Categories of deltaF508 homozygous cystic fibrosis twin and sibling pairs with distinct phenotypic characteristics. Twin Res 2000;3: 277-293.
28 Bronsveld I, Mekus F, Bijman J, et al: Residual chloride secretion in intestinal tissue of deltaF508 homozygous twins and siblings with cystic fibrosis. The European CF Twin and Sibling Study Consortium. Gastroenterology 2000;119:32-40.

29 Bronsveld I, Mekus F, Bijman J, et al: Chloride conductance and genetic background modulate the cystic fibrosis phenotype of Delta F508 homozygous twins and siblings. J Clin Invest 2001;108:1705-1715.

30 Mekus F, Laabs U, Veeze H, Tummler B: Genes in the vicinity of CFTR modulate the cystic fibrosis phenotype in highly concordant or discordant F508del homozygous sib pairs. Hum Genet 2003;112:1-11.

-31 Drumm ML, Konstan MW, Schluchter MD, et al: Genetic modifiers of lung disease in cystic fibrosis. N Engl J Med 2005;353:14431453.

32 Pulleyn LJ, Newton R, Adcock IM, Barnes PJ: TGFbeta1 allele association with asthma severity. Hum Genet 2001;109:623-627.

-33 Wu L, Chau J, Young RP, Pokorny V, et al: Transforming growth factor-betal genotype and susceptibility to chronic obstructive pulmonary disease. Thorax 2004;59:126129.

34 Silverman ES, Palmer LJ, Subramaniam V, et al: Transforming growth factor-betal promoter polymorphism C-509T is associated with asthma. Am J Respir Crit Care Med 2004;169:214-219.

35 Celedon JC, Lange C, Raby BA, et al: The transforming growth factor-betal (TGFB1) gene is associated with chronic obstructive pulmonary disease (COPD). Hum Mol Genet 2004;13:1649-1656.

36 Hull J, Thomson AH: Contribution of genetic factors other than CFTR to disease severity in cystic fibrosis. Thorax 1998;53:10181021.

37 Davies JC, Turner MW, Klein N: Impaired pulmonary status in cystic fibrosis adults with two mutated MBL-2 alleles. Eur Respir J 2004;24:798-804.

38 Yarden J, Radojkovic D, De Boeck K, et al: Association of tumour necrosis factor alpha variants with the CF pulmonary phenotype. Thorax 2005;60:320-325.

-39 Garred P, Pressler T, Madsen HO, et al: Association of mannose-binding lectin gene heterogeneity with severity of lung disease and survival in cystic fibrosis. J Clin Invest 1999;104:431-437.

40 Arkwright PD, Laurie S, Super M, et al: TGFbeta(1) genotype and accelerated decline in lung function of patients with cystic fibrosis. Thorax 2000;55:459-462.

41 Davies JC, Griesenbach U, Alton E: Modifier genes in cystic fibrosis. Pediatr Pulmonol 2005;39:383-391. 
42 Cutting GR: Modifier genetics: cystic fibrosis. Annu Rev Genomics Hum Genet 2005;6 237-260.

43 Zielenski J, Corey M, Rozmahel R, et al: Detection of a cystic fibrosis modifier locus for meconium ileus on human chromosome 19q13. Nat Genet 1999;22:128-129.

44 Rubin BK: Exposure of children with cystic fibrosis to environmental tobacco smoke. N Engl J Med 1990;323:782-788.

45 Kovesi T, Corey M, Levison H: Passive smoking and lung function in cystic fibrosis. Am Rev Respir Dis 1993;148:1266-1271.

-46 Smyth A, O’Hea U, Williams G, et al: Passive smoking and impaired lung function in cystic fibrosis. Arch Dis Child 1994;71:353354.

-47 Shepherd R, Cooksley WG, Cooke WD: Improved growth and clinical, nutritional, and respiratory changes in response to nutritional therapy in cystic fibrosis. J Pediatr 1980; 97:351-357.
48 Dalzell AM, Shepherd RW, Dean B, et al: Nutritional rehabilitation in cystic fibrosis: a 5 year follow-up study. J Pediatr Gastroenterol Nutr 1992;15:141-145.

49 Steinkamp G, von der Hardt H: Improvement of nutritional status and lung function after long-term nocturnal gastrostomy feedings in cystic fibrosis. J Pediatr 1994;124: 244-249.

50 Zemel BS, Jawad AF, Fitzsimmons S, Stallings VA: Longitudinal relationship among growth, nutritional status, and pulmonary function in children with cystic fibrosis: analysis of the cystic fibrosis foundation national CF patient registry. J Pediatr 2000;137: 374-380.

51 Kirvela O, Stern RC, Askanazi J, et al: Longterm parenteral nutrition in cystic fibrosis. Nutrition 1993;9:119-126.

52 John M, Ecclestone E, Hunter E, et al: Epidemiology of Pseudomonas cepacia colonization among patients with cystic fibrosis. Pediatr Pulmonol 1994;18:108-113.
53 Farrell PM, Shen G, Splaingard M, et al: Acquisition of Pseudomonas aeruginosa in children with cystic fibrosis. Pediatrics 1997; 100:E2.

54 Kerem E, Corey M, Gold R, Levison H: Pulmonary function and clinical course in patients with $\mathrm{CF}$ after pulmonary colonization with Pseudomonas aeruginosa. J Pediatr 1990;116:714-719.

55 Demko CA, Byard PJ, Davis PB: Gender differences in cystic fibrosis: Pseudomonas aeruginosa infection. J Clin Epidemiol 1995;48: 1041-1049.

56 Corey M, Farewell V: Determinants of mortality from cystic fibrosis in Canada, 19701989. Am J Epidemiol 1996;143:10071017.

57 Parad RB, Gerard CJ, Zurakowski D, et al: Pulmonary outcome in cystic fibrosis is influenced primarily by mucoid Pseudomonas aeruginosa infection and immune status and only modestly by genotype. Infect Immun 1999;67:4744-4750. 